\title{
El Repertorio Americano (1974-1983): primera revista académica fundada en la Universidad Nacional de Costa Rica
}

\author{
Marybel Soto Ramírez ${ }^{1}$ \\ Universidad Nacional de Costa Rica (Costa Rica) \\ marybel.soto.ramirez@una.cr
}

Recepción: 12/05/2012

Evaluación: 04/07/2012

Aceptación: 03/11/2012

Artículo de Reflexión

DOI: http://dx.doi.org/ 10.9757/Rhela.20.07

\section{RESUMEN}

El artículo presenta hallazgos de la tercera época del Repertorio Americano, iniciada en 1974 cuando la Revista y la valiosa colección de García Monge, publicada de 1919 a 1958, pasaron a formar parte del acervo intelectual de una nueva universidad costarricense que nacía con un planteamiento político y educativo característico. La Universidad Nacional de Costa Rica (UNA), gestada como la Universidad Necesaria, se comprometió a volver a publicar Repertorio Americano como órgano de difusión intelectual y vinculación académica con proyección latinoamericanista. Metodológicamente la labor de archivo permitió localizar y compilar documentos histórico-legales e institucionales para analizar el proceso de planteamiento, justificación y negociación para la cesión de derechos de propiedad intelectual del impreso complementariamente con la realización de entrevistas a uno de los miembros de la comisión ad-hoc organizadora de la Universidad. Para analizar la Revista como objeto de estudio se recurrió al análisis funcional del impreso.
Los resultados permiten concluir que Repertorio Americano en la UNA no fue un hecho fortuito dentro el proceso de creación y gestión de la naciente Universidad. Se constata la confluencia de factores político-culturales relacionados con el papel que asumía la Universidad Necesaria imbuida por los postulados del pensamiento latinoamericano de la década de 1970. Finalmente, se puntualizan los esfuerzos del primer comité de redacción al definir y organizar editorialmente Repertorio Americano como revista universitaria a partir de los puntos de contacto y diferencia con su predecesora con la cual mantiene el ideal de acercamiento de los pueblos por la cultura y la educación.

Palabras clave: Revista Historia de la Educación Latinoamericana, Repertorio Americano, Universidad Nacional de Costa Rica, publicaciones periódicas, revistas académicas, política editorial.

1 Doctorante del Programa en Estudios Latinoamericanos con énfasis en Pensamiento Latinoamericano de la Universidad Nacional de Costa Rica. Académica e investigadora del Instituto de Estudios Latinoamericanos, IDELA, donde coordina el Programa Integrado Repertorio Americano. Docente en los cursos de Historia de las Ideas en América Latina, Pensamiento Latinoamericano y Literatura, pensamiento y sociedad en América Latina de la Universidad Nacional de Costa Rica. 


\author{
The Repertorio Americano (1974-1983): first \\ academic journal founded in the National \\ University of Costa Rica
}

\author{
Repertorio Americano (1974-1983): primeira \\ revista acadêmica fundada na Universidade \\ Nacional de Costa Rica
}

\begin{abstract}
The article presents findings of the third epoch of the Repertorio Americano initiated in 1974 when the journal and the valuable collection of Garcia Monge that was published from 1919 to 1958, happened to be a part of the intellectual undivided assets of a new Costa Rican university that was born with a typical politic and educational proposal. The National University of Costa Rica, developed as a Required University, promised to publish again American Repertoire as an organ of intellectual diffusion and academic link with projection latinamericanism.
\end{abstract}

Methodologically the work file had allowed locating and compiling documents historicallegal and institutional to analyze the approach process, justification and negotiation for the assignment of intellectual property rights of the print in complement with the interviews with one of the members of the ad-hoc commission organizer of the University. To analyze the magazine as an object of study was employed to the functional analysis of the form.

The results allow the conclusion that American Repertoire in the UNA was not a fortuitous event within the process of creation and management of the nascent university. There is a confluence of facts politic-cultural related to the role that assumed the Required University imbued with the Latin American thought of the 1970s. Finally, we point out the efforts of the first editorial board to define and organize American Repertory editorially as university magazine from the points of contact and difference from its predecessor with which maintains the ideal of bringing peoples to culture and education.

Key Words: History of Latin American Education Journal, Repertotio Americano, Universidad $\mathrm{Na}$ cional de Costa Rica, regular publishings, academic journals, editorial policy.

\section{RESUMO}

O artigo apresenta achados da terceira época do Repertorio Americano, iniciada em 1974, quando a revista e a valiosa coleção de García Monge, publicada de 1919 a 1958, passaram a fazer parte do acervo intelectual de uma nova universidade costarriquense que nascia com um projeto político e educativo característico. A Universidade Nacional de Costa Rica, gestada como a Universidade Necessária, se comprometeu a voltar a publicar Repertorio Americano como órgão de difusão intelectual e vinculação acadêmica com projeção latinoamericanista.

Metodologicamente, o trabalho de arquivo permitiu localizar e compilar documentos histórico-legais e institucionais para analisar o processo de apresentação, justificação e negociação para a cessão de direitos de propriedade intelectual do impresso complementarmente com a realização de entrevistas a um dos membros da comissão adhoc organizadora da Universidade. Para analisar a revista como objeto de estudo recorreu-se à análise funcional do impresso. Os resultados permitem concluir que Repertorio Americano, na UNA, não foi um fato fortuito dentro do processo de criação e gestão da nascente universidade. Constata-se a confluência de fatores político-culturais com o papel que assumia a universidade necessária, imbuída dos postulados do pensamento latino-americano da década de 1970. Finalmente, são pontuados os esforços do primeiro comitê de redação ao definir e organizar editorialmente Repertorio Americano como revista universitária a partir dos pontos de contato e diferença com sua predecessora com a qual mantém o ideal de aproximação dos povos pela cultura e pela educação.

Palavras-chave: Revista História da Educação Latino-americana,Repertorio Americano, Universidade Nacional de Costa Rica, publicações periódicas, revistas acadêmicas, política editorial. 


\section{INTRODUCCIÓN}

Este artículo es uno de los resultados del proyecto de investigación titulado Repertorio Americano en la Universidad Nacional: rescatando la memoria de una misión histórica e intelectual (1974-1983) inscrito en el Programa Integrado Repertorio Americano, del Instituto de Estudios Latinoamericanos, IDELA (aprobado por la Vicerrectoría de Investigación, código SIA 0176-10).

Es posible establecer tres etapas editoriales en la perspectiva histórica de la Revista Repertorio: en 1826, dirigida por don Andrés Bello, un segundo momento, que abarca de 1919 a 1958, cuando el impreso es editado y dirigido por don Joaquín García Monge quien abiertamente define que esta etapa tiene en el Repertorio de Bello un ideal y un ejemplo a seguir; y el tercer momento, cuando a partir de 1974 los derechos de publicación de la revista de Joaquín García Monge fueron cedidos a la Universidad Nacional de Costa Rica. ${ }^{2}$

La relevancia de este estudio se justifica en el hecho de que en 1974 Repertorio Americano surge nuevamente a la luz, pero esta vez como órgano de cátedra para la difusión intelectual y vinculación académica aunque manteniendo siempre su proyección latinoamericanista. De tal forma, se comprende además la importancia de este análisis en que con la propuesta de publicación de la Revista por la UNA, Repertorio se convierte en la primera publicación académica fundada en la nueva universidad, inscrita en el quehacer académico del Instituto de Estudios Latinoamericanos (IDELA).

¿Cómo se enmarcó su publicación en el contexto del nacimiento de la universidad como proyecto educativo imbuido del pensamiento latinoamericano del decenio? ¿Quiénes fueron los propulsores de la idea de volver a publicar la Revista? ¿Quiénes ayudaron a consolidarla editorialmente? son preguntas clave que guiaron esta investigación.

Para despejarlas metodológicamente se buscaron, identificaron y analizaron los 34 números de Repertorio Americano que componen su tercera época, correspondientes al período 1974-1983.

2 Marybel Soto Ramírez, "El Repertorio Americano en perspectiva histórica. Roberto Brenes Mesén en el Repertorio Americano: contribuciones al pensamiento político costarricense" (Tesis de Licenciatura en Estudios Latinoamericanos. Universidad Nacional de Costa Rica, 1998). 
Se realizó una exhaustiva labor de investigación de archivos para lo cual se recurrió, entre otros, al Sistema de Archivo Institucional de la UNA y al Archivo Nacional para localizar, identificar, compilar y analizar documentos históricos, legales, oficiales e institucionales con el fin de averiguar qué procesos y negociaciones fueron realizados para lograr la cesión de derechos intelectuales de la Revista.

Aunque el trabajo se basa fundamentalmente en análisis documental, se logró entrevistar ${ }^{3}$ a uno de los primordiales gestores de la idea de publicar Repertorio Americano, y miembro clave de la comisión ad-hoc organizadora de la UNA, lo cual permitió descubrir detalles pormenorizados de los planteamientos político-culturales asumidos por la UNA en sus momentos genesiacos para así poner en perspectiva los planteamientos y la decisión para revivir Repertorio Americano.

Finalmente, para establecer un perfil editorial de la tercera época, se realizó un análisis funcional del impreso, tomando en consideración los aspectos de cercanía y diferencia con el Repertorio de García Monge y se sistematizó cómo fueron los primeros años de definición y consolidación editorial de la primera publicación académica fundada en la Universidad Nacional.

En términos metodológicos, la propuesta se sustenta en el planteamiento de que la revista académica pasa de ser fuente (científica, académica e institucional de difusión) a objeto de estudio. La revista, como documento histórico, permite rastrear, identificar y sistematizar experiencias. De tal forma, una revista académica encierra un universo en sí misma, donde los procesos de edición, impresión, circulación, lectores, políticas de publicación y distribución pueden dar cuenta de formas de producción y circulación del conocimiento, de los paradigmas políticos, educativos, científicos y culturales, de las formas de expresión y validación del canon científico que rigen no solo a la revista sino que conforman, y a la vez modelan, a la comunidad intelectual que da vida a la publicación.

La importancia que juega la investigación de las publicaciones como medio de difusión cultural, científica, literaria en América Latina está sólidamente

3 No es objetivo de este trabajo ofrecer los resultados de reconstrucción a partir de una fuente oral, aspecto que se espera proponer en otro momento investigativo, mediante la estrategia metodológica de sistematización de experiencias. 
documentada. ${ }^{4}$ Los impresos se estudian con el fin de rastrear y establecer con claridad el pensamiento de un autor, la política editorial que anima a la publicación editorial, los circuitos de producción, las redes y círculos de lectores que se establecen alrededor del impreso.

También es posible investigar el aspecto histórico donde surge y se desarrolla la Revista, lo cual permite valorar y dar seguimiento al contexto global de creación y desarrollo del impreso de interés y a las relaciones políticas, culturales y de sociabilidad intelectual que se gestan y se establecen en determinado momento de su vida o a lo largo de la existencia de una revista. A esto es a lo que el señalamiento metodológico de la investigadora argentino-mexicana Alexandra Pita $^{5}$ denomina el paso de la revista de fuente a objeto de estudio.

Pita $^{6}$ establece que cuando se realiza una intervención de análisis en las revistas, para estudiarlas, pasan de ser fuentes (de divulgación académica) a objetos de estudio, es decir, objetos de investigación, susceptibles de analizarse - en sí mismas y por sí mismas- mediante diversas llaves de acceso: desde el análisis de contenido y el análisis textual e impactos bibliométricos, hasta la investigación y análisis de historia de las ideas, con lo cual se posibilita trazar itinerarios eidéticos, intelectuales, ideológicos, tanto de la revista en sí, como de las personas participantes, de los circuitos de producción y recepción y del campo cultural. El primer paso es construir una cartografía del impreso a partir del contexto global de creación de la revista y de su perfil funcional.

En términos metodológicos y de aporte al conocimiento, la tercera salida del Repertorio Americano como objeto de estudio es relevante y pertinente porque permite recuperar, a partir del estudio de las condiciones de producción de esta Revista, la memoria histórica de una gesta intelectual que significó la idea de revivir la publicación de Repertorio Americano como vehículo primo de divulgación en el contexto de la Universidad Nacional de Costa Rica

4 Alexandra Pita, "Revistas culturales y redes intelectuales: Una aproximación metodológica”. Ponencia presentada en el Coloquio Internacional La Revista Politica en América Latina: el compromiso de la intelectualidad (Heredia: 2012) Material en prensa - 2012; Alexandra Pita y Carlos Marichal, coords. Pensar el antiimperialismo, Ensayos de historia intelectual latinoamericana, 1900-1930 (México: Colmex, Univesidad de Colima, 2012); Flora Ovares, “Crónicas de lo efímero", Revistas literarias de Costa Rica (2011): 356; Regina Crespo, coord. Revistas em América Latina: proyectos literários, políticos y culturales (México: CIALC, 2010); Mario Oliva, España desde lejos (Costa Rica: EUNED, 2011); Fernanda Galina Russell, Los nuevos retos de las revistas digitales académicas (Costa Rica: Proyecto Latindex, 2010); Fernanda Beigel, "Las revistas culturales como documentos de la historia latinoamericana en Utopía y Praxis, (Venezuela: LUZ, 2003), entre otros.

5 Pita, "Revistas".

6 Pita, "Revistas". 
(creada mediante la Ley 5182, del 15 de febrero de 1973). Dicha gesta intelectual refiere a los esfuerzos y a los gestores que llevaron adelante esa idea hasta concretarla. Segundo, el analizar la Revista permite la identificación de las líneas de política editorial que asistieron al impreso en sus primeros años de existencia, y tercero, trazar la identificación de los grandes ejes temáticos y de participación intelectual en la Revista.

Estos tres aspectos constituyen el diseño de un mapa cartográfico del que nos habla la metodología propuesta por Alexandra Pita para el análisis de las revistas y que expondremos más adelante.

\section{Repertorio Americano en el contexto histórico del proyecto político-educativo de la UNA}

Cuando se habla de Repertorio Americano se reconoce por derecho propio la obra de Joaquín García Monge. García Monge establece la relación de su Repertorio con el de de Andrés Bello, cuando dice que el suyo tiene en aquél "...una tradición respetable, un ejemplo y una guía que seguir". ${ }^{7}$

De similar manera, la primera fuente documental identificada en la investigación que habla sobre los esfuerzos de publicar nuevamente el Repertorio Americano, que había cerrado sus páginas con el fallecimiento de García Monge, en octubre de 1958, es el número primero de la denominada tercera época del Repertorio Americano, el correspondiente a octubre-diciembre de 1974. En esta edición, el intelectual costarricense Isaac Felipe Azofeifa publica un texto, a modo de editorial, titulado Tercera salida del Repertorio, donde hace una reflexión sobre el nombre de la Revista en las letras del continente y de la obra de cultura y periodismo de Joaquín García Monge:

El Repertorio Americano es un nombre venerable en las letras de nuestro continente. Andrés Bello lo creó en Londres en 1826. Después de los primeros cuatro números ese título quedó suspendido en el aire como una bandera, como un reto. Un siglo después, el oscuro maestro de un todavía más oscuro país de Centroamérica recogió el desafío y con el nombre otra vez de Repertorio Americano lanzó un cuadernillo mensual de escasas veinte páginas. (...) Veinticinco años más tarde, Repertorio Americano (...) recorría los caminos en todas direcciones, repartiendo la voz de España y de América por el mundo. El último

7 Joaquín García Monge, "El Otro Repertorio", Repertorio Americano, Vol.: VIII, No. 1 (1924): 39. 
día de octubre de 1958 murió aquel singular director del Repertorio. ¿Querría don Joaquín que su obra muriese con él? Todo lo contrario... ${ }^{8}$

El escritor Azofeifa señala los puntos de contacto entre la obra de García Monge y la de Bello, y expone que el Repertorio Americano en su tercera salida, como Revista universitaria, recoge la invitación formulada por García Monge: “¡Hay tarea para tantos, si quisieran trabajar! ¡Hay mucho que hacer todavía!". ${ }^{9}$ El Prof. Azofeifa ofrece como justificación para responder a la invitación la creación de la Cátedra García Monge en el Instituto de Estudios Latinoamericanos y la obtención de derechos de los herederos de García Monge para poner a circular de nuevo la Revista.

Sin embargo, el Prof. Azofeifa cuidadosamente establece la distancia que hay entre una y otra revista: “Ni don Joaquín pretendió igualar o imitar a Bello... ni nosotros pretendemos igualar a don Joaquín. Cada cosa en su tiempo", y al hacerlo establece las líneas generales de su programa editorial: "Nuestro Repertorio se publicará como cuadernos, en el mismo formato del de García Monge y ahí termina la semejanza". ${ }^{10}$

De esta forma, queda perfilada la identidad de la nueva Revista, que si bien no es la de García Monge, recoge su herencia de proyección cultural, humanística y latinoamericanista: "Echamos hoy a andar de nuevo la Revista con el mismo nombre, y con los mismos propósitos generales que animaron la obra, primero de Bello, y de García Monge después". ${ }^{11}$

Los miembros del consejo de redacción, los codirectores y los gestores de la idea de revivir Repertorio Americano tenían claridad sobre el programa editorial de la nueva Revista, que si bien recogía el ideal latinoamericano y cultural de don Joaquín, era diferente a la Revista editada por él, porque el Repertorio Americano en la UNA respondía a otro contexto histórico y de producción:

Nuestro tiempo tiene otro signo. Las revistas ya no son obra de una sola persona, sino de una institución, un circulo especializado. Una empresa publicitaria. En esta tercera etapa, Repertorio americano responderá

\footnotetext{
Isaac Felipe Azofeifa, “Tercera salida del "Repertorio", Repertorio Americano. Vol.: 1. No. 1 (1974): 3.

Isaac Felipe Azofeifa, "Tercera", 3.

10 Isaac Felipe Azofeifa, "Tercera"

11 Isaac Felipe Azofeifa, "Tercera"
} 
al propósito de órgano de una Cátedra y publicación de un Instituto Universitario: el tema americano que vivió en la pluma de pensadores, hoy es objeto de las ciencias sociales, políticas, económicas, literarias. ${ }^{12}$

En ese mismo número, se ofrece una exposición precisa de cómo surge la idea de publicación de la Revista, en una entrevista conjunta que se hizo al primer Rector de la Universidad, Pbro. Benjamín Núñez; al Lic. Francisco Morales Hernández, Ministro de Trabajo, miembro de la comisión ad-hoc, impulsor y gestor de la idea de la publicación, y al Dr. Chéster Zelaya, primer director del Instituto de Estudios Latinoamericanos, unidad académica donde se adscribió el Repertorio Americano:

A la Universidad Nacional, apenas nacida, se le han presentado muchos retos. Uno de ellos ha sido el de promover la publicación del Repertorio Americano, que fue en su tiempo antorcha alimentada por espíritus inquietos en las manos proféticas de Don Joaquín García Monge. Esta iniciativa fue concebida y alentada con entusiasmo por el Señor Ministro de Trabajo, Don Francisco Morales y acogida con aplauso por la comisión Organizadora de la Universidad Nacional. ${ }^{13}$

Luego de este número, la información sobre la tercera salida de Repertorio es prácticamente nula, a excepción de un artículo publicado en el diario $\mathrm{La}$ Nación, al celebrar el primer quinquenio de la nueva etapa de Repertorio y que, en lo fundamental, glosaba la información publicada en el número primero.

Para comprender el surgimiento y puesta en circulación de Repertorio Americano en su tercera etapa, se requiere comprender el contexto histórico inmediato que refiere al surgimiento de la Universidad Nacional de Costa Rica, como proyecto político educativo revolucionario, ${ }^{14}$ así como a los paradigmas filosóficos y sociales que la nutren en el contexto del pensamiento latinoamericano.

12 Isaac Felipe Azofeifa, “Tercera”, 4.

13 Benjamín Núñez, “Por qué Repertorio Americano en la Universidad Nacional”, Repertorio Americano, Vol.: 1 No. 1 (1974): 7.

14 Así lo califica el Lic. Francisco Morales en entrevista con la investigadora en enero 2013. 


\section{La Universidad Nacional como Universidad Necesaria: dimensiones políticas de un proyecto educativo}

La Universidad Nacional de Costa Rica fue creada mediante la Ley 5182 del 15 de febrero de $1973,{ }^{15}$ y, para organizarla administrativa y académicamente, a los ocho días de aprobada la Ley, el consejo de gobierno nombró una comisión $a d-h c^{16}$ liderada por el Presbítero Dr. Benjamín Núñez Vargas y compuesta por los ministros de educación, Lic. Uladislao Gámez; de planificación económica, Lic. Oscar Arias Sánchez y de trabajo, Lic. Francisco Morales; además de un selecto grupo de académicos de la Universidad de Costa Rica con rango de catedráticos asociados.

La creación de la Universidad nueva, como lo calificaba el Dr. Núñez Vargas, se asumiría desde una posición particular contrastante con la de la universidad tradicional en lo que el historiador Araya Pochet identifica como "... un modelo académico novedoso, orientado en la filosofía latinoamericana de Universidad necesaria..." 17 donde explícitamente, y de acuerdo con el Estatuto Orgánico de 1976: “La investigación constituye un elemento central de las actividades universitarias. Alimenta la docencia y la extensión al diagnosticar la realidad nacional, al proponer nuevas alternativas de desarrollo y plasmarlas en acciones académicas estratégicas". ${ }^{18}$

En el pensamiento del Dr. Núñez Vargas, primer rector de la UNA, el adjetivo necesaria incluía lo que él denominaba una internacionalización, y aunque advertía sobre la inexistencia del vocablo le confería significado a partir de la vocación de la Universidad y de sus fines: "orientar la tarea de producir conocimiento permanente hacia la interpretación de la realidad de una sociedad determinada...". ${ }^{19}$ La excelencia académica como primer compromiso daba sentido a la universidad, pero para el Rector Núñez había también un sentido trascendental cifrado en el compromiso de la nueva universidad con la transformación social mediante la "contribución efectiva a un desarrollo

15 Costa Rica, Asamblea Legislativa de la República de Costa Rica, Ley de creación de la Universidad Nacional, ley de la República, No. 5182, 15 de febrero de 1973 País, organismo que dicta la norma y clase, número y fecha de la norma. <www.pgr.go.cr> (consultada el 13 de octubre de 2012).

16 Esta comisión, dirigida por el Pbro. Núñez, cumplió las labores de Consejo y Asamblea universitarias y sesionó por dos años, hasta la instauración, del primer consejo universitario de la UNA, el 25 de febrero de 1975.

17 Carlos Araya Pochet, La Universidad Nacional y la educación superior estatal en veinte años de historia (1973-1993). (Hereda: EUNA, 1993), 53.

18 Araya, "La Universidad", 52.

19 Benjamín Núñez, Hacia la Universidad Necesaria (Heredia: Dpto. de Publicaciones, Universidad Nacional, 1974), 9. 
equilibrado y autónomo de la sociedad". ${ }^{20}$ Se planteaba, de esta manera, el diálogo con la sociedad para aprehender de ella y transformarla, dialécticamente, con criterios de racionalidad y verdad, prosperidad, justicia y libertad, como justificaba filosóficamente la función de la universidad. ${ }^{21}$

Es decir, que más allá de la necesidad del pueblo por una nueva institución de educación superior, el proyecto de modelo organizativo presupuso un contenido en lo educativo y lo político, fundado en una intención de cambio y mejoramiento, alimentada en las ideas del pensamiento latinoamericano de la década de 1970, en particular en pensadores como el sociólogo Darcy Riveiro, para quien ciencia, técnica y humanismo, debían hacerse coincidir para el avance de la educación superior en Nuestra América.

La Universidad Necesaria, la universidad nueva, planteaba su quehacer internacionalizado como el de un ente vivo y en construcción, en estrecha comunicación con la realidad social del país, no solo para su estudio sino para su transformación, un quehacer "orientado hacia objetivos que deben ser claramente expresados", ${ }^{22}$ abanderando así el ideal de compromiso social, renovación de la educación superior y democratización educativa y de la sociedad, que recorría los ideales de la geografía latinoamericana. El Estatuto Orgánico de 1976 declara: “...la Universidad Nacional dirige su principal quehacer a la eliminación del subdesarrollo". ${ }^{23}$

En consecuencia, la misión de la Universidad se definiría no solo como un posicionamiento epistemológico sino también político en la concreción de la racionalidad organizativa de la institución, como ente necesario a la sociedad costarricense y que asume su vocación, según se desprende de los planteamientos de su Estatuto Orgánico de 1976, en la transformación social y profundización de la democracia mediante la participación de los sectores populares y en ser facilitadora para que estos sectores populares tuvieran acceso al disfrute de los bienes culturales y el mejoramiento material.

20 Benjamín Núñez, “Una universidad de excelencia. Universidad Nacional”, en Historia y utopía, compilado por Olmedo España Calderón (Heredia: Editorial Fundación UNA, 1998): 58.

21 Celedonio Ramírez, "La función de la nueva universidad”, Repertorio Americano, Vol.: 1, Año. 2 (1975): 2.

22 Benjamín Núñez, "Hacia la Universidad", 10.

23 Universidad Nacional, Consejo Universitario, Estatuto Orgánico, 1976 (Heredia: Depto. de Publicaciones), 4. 


\section{Repertorio Americano en la UNA: bases de una misión histórica e intelectual}

Como se comprueba con lo anteriormente expuesto, la Universidad Nacional nació con un ideal alimentado en las fuentes del pensamiento liberador de la Universidad Necesaria latinoamericana, que promovía la participación y formación de los sectores menos favorecidos del desarrollo nacional, de la "consciencia de transformación social y reforma universitaria" ${ }^{24}$ que existía en Costa Rica en la década de 1970, en el sentido de profundización de las condiciones de autonomía y libertad, y en el compromiso de mejoramiento social y democrático hacia la superación del subdesarrollo.

Se establecería como su función sustantiva la directa relación de la investigación y el desarrollo, la participación universitaria en los planes nacionales de desarrollo, la mejoría de las condiciones sociales de los costarricenses y la ampliación de la participación de las mayorías, así como dar posibilidad a la formación de profesionales en concordancia con las exigencias de desarrollo del país y no solamente en términos de mercado.

El papel de la cultura y del humanismo se consideró medular en el proyecto de la universidad necesaria. Una cultura, no elitista ni degradada para el consumo de las masas sino una cultura liberadora, dignificadora, en su más amplia acepción, de la condición humana.

En este ambiente humanista, comprometido, científico y heredero del pensamiento intelectual generado por la Escuela Normal Omar Dengo, con un posicionamiento claro sobre la cultura y la problemática social, el Lic. Francisco Morales Hernández, en su condición de Ministro de Trabajo y miembro de la comisión ad-hoc organizadora de la Universidad Nacional, presentó la idea de revivir en ese nuevo contexto académico el Repertorio Americano, que había dejado de publicarse en 1958, como un órgano de cultura con proyección latinoamericanista.

La misión histórica de volver a poner en circulación Repertorio Americano, alcanzaba pleno significado y sentido en palabras del Rector Núñez quien establecía en: “...una Universidad que no quiere ser torre de marfil sino

24 Núñez, "Una universidad", 83. 
empresa científica, académica y cultural, vibrando con la realidad nacional y sirviendo a un pueblo impaciente por su liberación integral" ${ }^{25}$

El primer documento oficial que por aspectos heurísticos debe citarse es el Acta número 55, de fecha 18 de setiembre de 1973. Esta acta es de gran valor porque ofrece un panorama muy acotado de cómo fueron organizándose las facultades y escuelas y de qué académicos se propone para dirigirlos. El punto 3.1.8. Establece la creación del Instituto de Estudios Latinoamericanos, definido como una unidad especializada, interdisciplinaria y de proyección internacional, en palabras del Dr. Rodrigo Zeledón, "integradora en los diversos campos de investigación latinoamericana" ${ }^{26}$ cuyo propósito es el de investigar y dar a conocer la cultura latinoamericana en todos los campos.

El Acta 68, del 29 de noviembre de 1973, es el primer documento que da cuenta de las intenciones de recoger el ideal de García Monge y de volver a publicar Repertorio Americano. En el artículo 5, el Lic. Francisco Morales formula la propuesta para que Instituto de Estudios Latinoamericanos lleve el nombre de Joaquín García Monge y justifica que: “La deuda de los costarricenses con don Joaquín García Monge y su Repertorio Americano es enorme y aún no ha sido saldada. (...) La Universidad Nacional podrá hacerse cargo de esta interesante labor y propone formalmente (sic) a la comisión se contemple designar con el nombre García Monge el programado Instituto de Estudios Latinoamericanos". ${ }^{27}$

La comisión ad-hoc y el Rector Núñez encomiendan al Lic. Morales llevar a cabo una serie de gestiones para tratar de lograr volver a publicar la Revista, entre ellas, sostener conversaciones con los herederos de los derechos del impreso. Las actas subsiguientes, hasta abril de 1974, van facilitando información sobre las acciones del Lic. Morales. De su propia voz, se recoge el siguiente testimonio:

Entonces, me puse en contacto con el Dr. Eugenio García Carrillo. Yo era Ministro de Trabajo, en ese momento. Le pedí una audiencia y lo invité al despacho del Ministro. Le dije: "Mire, doctor, estamos en este

25 Núñez, "Por qué Repertorio", 8.

26 Actas Comisión Organizadora AD-HOC. Sesión No. 55 (Heredia, 18 de setiembre de 1973) Acta No. 55 Comisión Ad-hoc, Heredia, Sistema Institucional de Archivos, Universidad Nacional, 6.

27 Actas Comisión Organizadora AD-HOC. Sesión No. 68. (Heredia, 29 de noviembre de 1973) Sistema Institucional de Archivo, Universidad Nacional (folio No. 0026). 
proyecto de crear una universidad y Don Lalo Gámez, el Padre Benjamín Núnez, Isaac Felipe Azofeifa, Rose Marie Karspinski, Francisco Antonio Pacheco, Roberto Murillo y yo, creemos que esta universidad, que nace en Heredia, constituyéndose con las Normales Superiores, nace en la vieja Normal de Heredia donde estuvieron los cuatro grandes intelectuales nuestros, Carlos Gagini, Roberto Brenes Mesén, -García Monge y Omar Dengo. En esa tierra abonada por los González Flores don Alfredo, el Presidente transformador y su hermano, gran educador, Luis Felipe-con alumnos de esos cuatro pensadores costarricenses como son mismo Ministro de Educación, don Lalo Gámez, como las maestras escritoras, Luisa González, Adela Ferreto, Emma Gamboa, como Corina Rodríguez, como León Pacheco, que viven y con quienes hemos conversado esta idea de crear la universidad, nos parece que la nueva Institución está comprometida a publicar el Repertorio Americano, como órgano oficial de la Universidad Nacional".

$Y$ eso impresionó mucho al Doctor y dijo: "Pues créame, Señor Ministro, que es una sorpresa gratísima para mí. ${ }^{28}$

De noviembre de 1973 al 2 de abril de 1974, las gestiones emprendidas por el Lic. Francisco Morales Hernández con el Dr. Eugenio García Carillo dieron resultado. El Dr. García Carrillo fue recibido en sesión solemne de la Comisión ad-hoc, según se desprende en el Acta 94:

El señor rector presenta al Dr. Eugenio García Carrillo, hijo del ilustre costarricense don Joaquín García Monge. A nombre de la Comisión Ad-hoc le da la más cordial bienvenida y le invita a participar de la sesión. Seguidamente el Ministro de Trabajo, Lic. Francisco Morales concreta las razones que lo indujeron a solicitar la presencia del Dr. García Carrillo en esta reunión y le cede la palabra. (...) El Lic. Morales mociona para que la UNA recoja la publicación de Repertorio Americano. (...) con la cesión de derechos de autor que el Dr. García Carrillo está anuente a realizar a favor de la Universidad Nacional y que hoy viene a concretar y ratificar. ${ }^{29}$

Ante las palabras de agradecimiento del Ministro de Educación, de su complacencia porque Repertorio vuelva a manos de jóvenes y de que será el máximo galardón de la Universidad Nacional, el Dr. García Carrillo expresa: “Es un momento de gran emoción. Estaba preocupado por volver a revivir

28 Entrevista a Morales Hernández, Francisco, Heredia, 22 de enero, 2013.

29 Comisión ad-hoc de la Universidad Nacional. Acta No. 94, Art. 4, (2 de abril de 1974), s/p. 
Repertorio Americano y con esta decisión de la Universidad Nacional, por estar vinculada a la Escuela Normal, hará posible su retorno a Heredia". ${ }^{30}$

En términos globales los documentos oficiales ofrecen una visión del proceso seguido en la Universidad para tender las líneas generales de consolidación de su proyecto educativo. En términos específicos, informan sobre el perfil del Instituto de Estudios Latinoamericanos como un instituto especializado e internacional y de su objeto inmediato de estudio, de su sentido interdisciplinario y de las posibilidades de investigación y conformación del cuerpo académico. Pero si se va más allá, se descubre el interés en rescatar el pensamiento de García Monge en la cultura latinoamericana en varios puntos; por ejemplo: la propuesta de que el Instituto llevara el nombre del Benemérito; la creación de una Cátedra denominada Joaquín García Monge; el depósito de la colección Repertorio Americano donada por la familia García en el Instituto y la publicación del Repertorio Americano por parte de la UNA inscrito en el quehacer académico del Instituto. Solo concatenando estos hechos a partir de los hallazgos de esta investigación, se comprende que la publicación de Repertorio Americano más que una decisión cultural y editorial encierra un contenido político.

Al interrogar los documentos históricos oficiales institucionales, en primera instancia es posible corroborar que los esfuerzos realizados para justificar teórica, cultural y políticamente la importancia de que la Universidad Nacional fuera el lugar donde Repertorio Americano volviera a la luz, señalan la voluntad y el interés del Lic. Morales en proponer la idea y la de los miembros de la Comisión ad-hoc en asumir ese compromiso. Segundo, la recopilación y estudio de la documentación oficial de la Universidad evidencia los contactos y las negociaciones realizadas por el Lic. Francisco Morales con los herederos de García Monge para revivir la publicación con el cuidado de los aspectos legales para la cesión de derechos de propiedad intelectual de la Revista. La idea de que la Revista podría volver a publicarse si se contaba con una infraestructura académica que fuera propicia o coincidente con perfil latinoamericano original de la Revista era coincidente con que la idea del Rector Núñez y la comisión ad-hoc de que Repertorio Americano sería un broche de oro como carta de presentación para la nueva Universidad. El sentido de que la Universidad Nacional no solo saldaría una deuda pendiente con la

30 Eugenio García Carrillo. Acta No. 94, Comisión Ad-hoc de La Universidad Nacional, SIA, 2 de abril de 1974, Art. 4. s/p. 
memoria del Benemérito sino que en razón de ser la Universidad Necesaria, establecía coincidencia con el ideal pedagógico, cultural, nacionalista y de justicia social enarbolado por Repertorio Americano y por García Monge.

Finalmente, la importancia de la ciudad de Heredia cuna de la nueva universidad, por haber sido escenario de desarrollo de la Escuela Normal que durante años agitó el pensamiento, la conciencia y la cultura nacionales, que había sido dirigida por García Monge y sus discípulos, reforzaba la justificación de que Repertorio Americano volviese a ser publicado ahora por la UNA. La Revista regresaba, a en cierta forma, a su origen.

En segunda instancia, se puedes concluir que Repertorio Americano es la primera revista institucional de la Universidad, tomando en consideración que la UNA echó a andar el 15 de marzo de 1973 y que el 29 de noviembre de ese mismo año, el Lic. Francisco Morales presentó la propuesta de volver a publicar el Repertorio Americano por lo que le fue encomendado formalmente iniciar gestiones de negociación y cesión de derechos.

En febrero de 1974, las negociaciones dieron fruto y el Dr. García Carrillo, recibido en la Comisión ad-hoc, simbólicamente hace la cesión de derechos de propiedad intelectual a la UNA acción que se legaliza formalmente mediante la firma de una escritura pública, en octubre de 1974, entre el Rector Núñez y el Dr. García Carrillo. En diciembre de 1974, empieza a circular el primer número de la Revista, es decir, Repertorio Americano se publicó a un año y nueve meses de que la Universidad Nacional iniciara labores.

La conclusión de que Repertorio Americano es la primera revista académica de la UNA se refuerza con el hecho de que el impreso empezaría a dar cuenta del surgimiento de otras publicaciones institucionales, nacidas en el seno de las recientemente creadas unidades académicas. Tres publicaciones académicas a las cuales Repertorio Americano da la bienvenida por su creación son: Revista de Geografía de América Central; Revista de Historia y Revista Letras.

Si se concuerda con Paul Ricoeur respecto a que los archivos albergan una huella documental, que no es solo física sino también social, en tanto que guarda algo que pertenece a toda una comunidad y que conforma el rastro de su memoria colectiva, se considera que el interrogar los archivos, huella física, material, escrita, de los documentos analizados y de la Revista misma 
es factible adentrarse a un lugar de la memoria donde la interrogación de la investigadora es absolutamente necesaria para la comprensión y valoración de los hechos ya que, como apunta Ricoeur ${ }^{31}$ “...el documento que duerme en los archivos no solo es mudo, es también huérfano, los testimonios que oculta se separaron de los autores que lo crearon y están sujetos a los cuidados de quien tiene la competencia para interrogarlos...".

Los documentos analizados hablan de ese paso de la Revista de fuente a objeto, y de una gestión intelectual realizada con la convicción de estar haciendo la historia dentro la historia de nuestra Universidad Nacional.

\section{Repertorio Americano: Un análisis funcional del impreso}

Durante el período 1974-1983, la dirección del impreso se mantuvo en la figura de dos co-directores: el escritor, diplomático y profesor, don Isaac Felipe Azofeifa Bolaños y la académica, filóloga y escritora, doña María Rosa de Bonilla. Los codirectores, ambos académicos universitarios, estrechamente ligados al mundo de las letras latinoamericanas, imprimirían su sello a la Revista, a la cual calificaron de cuaderno y definieron en su orientación latinoamericanista. Lo literario está fuertemente arraigado en el impreso, como se comprueba en los más de 400 artículos publicados en el período.

La Revista definió una periodicidad trimestral, y estableció un consejo de redacción formado por académicos de diferentes áreas: Dr. Rolando Mendoza, biólogo, Lic. Carlos E. Aguirre, filólogo y abogado, la escritora Julieta Pinto, Directora de la Escuela de Literatura y Ciencias del Lenguaje; el Dr. Chéster Zelaya, historiador, director del IDELA, y el Lic. Carlos Ramírez.

En vista de que las etapas no son solo una referencia a cambios en el director o editor, sino también a diversidad en el tono discursivo y a los intereses del momento político o histórico, hay algunos elementos que vale la pena anotar. Se encuentra un primer sobresalto en la periodicidad, de octubre de 1976 a setiembre de 1977, cuando se publicó un número especial para este período de casi un año. Es factible pensar que un suceso mayor, fuera de las posibilidades de los codirectores, impidió que la Revista continuara con su salida trimestral en ese lapso. Por eso, el número especial que cubre este año

31 Paul Ricoeur, La memoria, la historia, el olvido (Madrid: Trota, 2003): 219. 
fue la opción para ponerse al día, para cumplir con las responsabilidades que se tenía con los autores, suscriptores y acuerdos de canje. El número especial es un ejemplar de mayor volumen que los regulares. Es importante indicar que a partir del número especial de octubre 1976 a setiembre 1977, empezó a introducirse fotografías, así como imágenes, siempre en blanco y negro, en la Revista y que se inicia la publicación de anuncios de actividades académicas nacionales e internacionales. No parece haber mediado cobro por la publicación de estos anuncios, sino que el hecho se explica por el medio académico en que se desarrolla el impreso, pues se trata de invitaciones a seminarios, a concursos literarios y ofrecimiento de becas.

Algunas características del aspecto funcional en contraste con el contexto global de surgimiento y desarrollo de la Revista se presentan en el siguiente cuadro. 


\section{Cuadro 1. Contexto global y contexto funcional de surgimiento de la Revista en su tercera salida}

\begin{tabular}{|c|c|}
\hline \multicolumn{2}{|c|}{ Surgimiento de Repertorio Americano en la UNA. } \\
\hline Contexto global de creación y desarrollo & $\begin{array}{l}\text { Contexto funcional de la revista } \\
\text { (Perfil) }\end{array}$ \\
\hline $\begin{array}{l}\text { Surge en el contexto inmediato de la } \\
\text { creación y forja de una universidad nueva, } \\
\text { cuyo perfil contrastaba con el } \\
\text { de la universidad tradicional. }\end{array}$ & $\begin{array}{l}\text { Surge con una función de ser órgano de } \\
\text { cultura y difusión (del pensamiento) en } \\
\text { el proyecto educativo de la Universidad } \\
\text { Nacional como universidad necesaria. }\end{array}$ \\
\hline $\begin{array}{l}\text { El contexto político latinoamericano de } \\
\text { 1970: luchas de liberación y revolución, } \\
\text { generalizada de la presencia militar en los } \\
\text { gobiernos. Idea del compromiso social por } \\
\text { el cambio y el mejoramiento. } \\
\text { El contexto histórico inmediato } \\
\text { costarricense, donde importantes } \\
\text { contingentes de población exigían } \\
\text { acceso a la educación superior }\end{array}$ & $\begin{array}{l}\text { Se asume desde el pensamiento } \\
\text { latinoamericano, desde la cultura y el } \\
\text { humanismo (teología y filosofía de la } \\
\text { liberación, literatura latinoamericana, } \\
\text { identidades, derechos humanos). } \\
\text { Preponderancia de las letras y la literatura. } \\
\text { Proyección regional/internacional. } \\
\text { Los jóvenes talentos de las letras } \\
\text { nacionales e internacionales encuentran } \\
\text { espacio en esta Revista. Así como los } \\
\text { jóvenes catedráticos universitarios. }\end{array}$ \\
\hline $\begin{array}{l}\text { Época de gran difusión de pensamiento } \\
\text { latinoamericano, autonomía, desarrollo, } \\
\text { solidaridad: Darcy Riveiro, Paulo Freire, } \\
\text { Ernesto Guevara } \\
\text { Luchas estudiantiles universitarias }\end{array}$ & $\begin{array}{l}\text { El derecho de Latinoamérica a hacerse oír. } \\
\text { Estética: nuevas tendencias literarias, } \\
\text { artísticas, pedagógicas, incluso } \\
\text { ambientales }\end{array}$ \\
\hline $\begin{array}{l}\text { Entorno internacional de la Guerra Fría. } \\
\text { Grandes hazañas de conquista del espacio } \\
\text { exterior. }\end{array}$ & $\begin{array}{l}\text { Acercamiento de los pueblos por su cultura } \\
\text { y por la educación. Derecho ético a la } \\
\text { autodeterminación de los pueblos. }\end{array}$ \\
\hline $\begin{array}{l}\text { La Universidad Nacional como } \\
\text { universidad latinoamericana, de convicción } \\
\text { popular y transformadora }\end{array}$ & $\begin{array}{l}\text { Publicado por el Instituto de Estudios } \\
\text { Latinoamericanos (que se propuso } \\
\text { llevaría el nombre del Maestro García } \\
\text { Monge), especializado, interdisciplinario } \\
\text { y de proyección internacional y } \\
\text { latinoamericanista. }\end{array}$ \\
\hline
\end{tabular}

Fuente: Sistematización propia a partir de los documentos consultados y la información recabada de los gestores del proyecto. 


\section{CONCLUSIÓN}

Repertorio Americano se desarrolla en un contexto donde las relaciones sociales que animaron su edición eran fundamentalmente académicas, instituidas por el proyecto pedagógico, político y cultural universitario. Esto es claro en la información que se ofrece desde en el primer ejemplar en 1974 sobre la Revista, al considerarse que su edición solo sería posible en un instituto interdisciplinario y especializado en América Latina, con lo cual se delimitaba también el campo de penetración de la Revista:

Tendrá que ser una labor moderna, de equipo, con sentido de quehacer interdisciplinario. Por eso, lo importante de que repertorio nazca y se desarrolle dentro del marco de un Instituto Latinoamericano, altamente especializado, donde se combinen-enriqueciéndose-los puntos de vista del filósofo, del literato, del economista, del sociólogo, del antropólogo $y$ del político. ${ }^{32}$

El programa editorial se centra en lo literario, en lo cultural latinoamericano y en el área del pensamiento. La insistencia de la invitación: “El Repertorio Americano, órgano al servicio de las letras y el pensamiento en América, le invita a formar parte de su grupo de amigos en varios puntos del mundo", 33 comprueba esta perspectiva.

En síntesis es posible cifrar los principios que fundamentan y dan guía al nuevo proyecto editorial de Repertorio de 1974 a 1983 en el espíritu latinoamericano que caracteriza la Revista, en su carácter de estar compuesta por misceláneas, lo que se mantiene como rasgo distintivo del Repertorio de García Monge, aunque esta variedad en la tercera época queda mucho más restringida por ser revista universitaria y órgano de cátedra; la identificación original de la Revista como un cuaderno de cultura se mantiene; la independencia ideológica y el pluralismo temático desde los cuales se organizó el proyecto editorial tanto garcía-mongiano como de la tercera época se profundiza y, finalmente, la investigación y la interdisciplinariedad que son pivote de su estructura editorial se fortalece al ser órgano universitario que se sustenta en la perspectiva académica para la difusión cultural y latinoamericana.

32 Francisco Morales. “Por qué Repertorio Americano en la Universidad Nacional”, Repertorio Americano Vol.: 1 No. 1 (1974): 8.

33 Repertorio Americano Año VI, No. 1 (1979): 30. 
Si bien el surgimiento y publicación de la tercera salida de en la Universidad Nacional buscaría perpetuar la memoria de don Joaquín y su producto editorial excelso, en la tercer etapa Repertorio Americano se institucionalizaría como un producto académico resultante del quehacer universitario que le concede características definitorias en su perfil. A partir de 1974 lograría el sentido de permanencia institucional que le concede ser una actividad académica con arraigo en las áreas de la investigación y de la extensión universitaria, formalmente formulada como un proyecto académico permanente del IDELA.

La red de colaboradores durante la tercera etapa se gestó originalmente por conocimiento personal de los dos primeros co-directores y de distinguidas personalidades del mundo cultural y de las letras costarricenses, y por contacto directo fue creciendo para abarcar a los jóvenes escritores y catedráticos universitarios, así como a intelectuales de las diferentes latitudes latinoamericanas. Las referencias que se empiezan a incluir de nombres de escritores y del tipo de colaboración que se buscaba captar, dan cuenta de esto: “...agradecemos con efusión cordial de latinoamericanos primero, de universitarios costarricenses juntamente, las colaboraciones que nos han enviado ya de los profesores: Eladio García, Hugo Montes y Juan Villegas, de Chile; Luis E. Aguilar, Cubano; Stefan Baciu, de Hawai; Claribel Alegría, de Mallorca". ${ }^{34}$

La red intelectual que se gesta alrededor de la revista está compuesta de personas asociadas a las letras latinoamericanas, mayormente escritores, académicos e investigadores, especialistas en literatura, estudiosos de la filosofía y en los primeros números, también por geógrafos y biólogos. Un total de 229 autores costarricenses y latinoamericanos, en menor medida europeos, fueron publicados en ese período. Entre los escritores se identificaron colaboraciones de: Manlio Argueta, de El Salvador; Claribel Alegría; de El Salvador; Oscar Héctor Pralong y Luis Ricardo Furlan, de Argentina; Juan Garavito, de Colombia; Teresinka Pereira, de Brasil, David Gross, de Estados Unidos, Julián Gustems, de España; Mario Monteforte Toledo, de Guatemala, Arturo Uslar Petri, de Venezuela, y los costarricenses: Quince Duncan; Carlos Rafael Duverrán, Lily Kruze, Leonor Garnier, Carlos Francisco Monge; Julieta Pinto; Alfredo Cardona Peña; León Pacheco, Adela

34 Isaac Felipe Azofeifa, "en este número", Repertorio Americano, Vol.: 2 No. 2, (1975): 33. 
Ferreto, Carlos Luis Sáenz, Alfonso Chase, Carmen Naranjo y Laureano Albán, entre otros.

Al lograr una cartografía básica del contexto histórico-funcional de la Revista es factible comprobar que Repertorio Americano en su tercera salida cumplió la función de órgano de divulgación cultural y de coadyuvar en la circulación de las ideas académicas latinoamericanas, en especial, referidas a la literatura, las artes, la educación y la pedagogía y el humanismo, tanto nacional como regionalmente, al publicar más de 500 artículos entre recensiones, ensayos, cuentos, lírica, relatos y discursos. Dejó, en contraste, la fuerte proyección de discusión política que identificaba el Repertorio de García Monge en América Latina. No claudica en ello totalmente por ese perfil propio de la Revista y del Instituto donde se inscribe y por el sentido latinoamericano y latinoamericanista, como se logra comprobar en las insistentes llamadas del profesor Azofeifa como co-director en la sección En este número, que fundamentalmente refería a la presentación y al editorial.

La política editorial se expresa en los objetivos de la Revista, definiéndola y dotándola con un contenido específico: así, ser ventana para mostrar lo que se produce en otros partes de Nuestra América; acercar por la cultura y la educación a los pueblos de nuestramérica, publicar avances de investigación y resultados de investigación, trabajos de reflexión y artículos sobre la realidad latinoamericana con perspectiva latinoamericanista, nos posa frente a una política editorial implícita en la producción y circulación de Repertorio Americano, a partir de su tercera salida.

El marco de relaciones que permite ofrecer una representación situacional del impreso ha permitido situar el surgimiento de la Revista como objeto en términos culturales, políticos e históricos para construir el itinerario del período comprendido de 1974 a 1983 de la primera revista académica de la Universidad Nacional de Costa Rica. Queda por investigar en detalle la participación de las personas colaboradoras de la Revista, como red intelectual incluida en una red cultural o académica mayor al señalar, brevemente por el momento, que cada edición constaba de una tirada 2000 ejemplares con los cuales se suplía el canje interbibliotecario y el envío a instituciones culturales y educativas latinoamericanas. 
Comprender Repertorio Americano en UNA como misión histórica e intelectual presentada a la comisión ad-hoc por el Lic. Francisco Morales y llevada adelante por la joven Universidad Nacional de Costa Rica solo es posible de valorarse en toda su dimensión al lograr dibujar la cartografía a partir de su surgimiento. El presente trabajo es el primer intento que ofrece una mirada, conforma un corpus y propone un inicio. Ojalá muchas nuevas investigaciones sobre el universo de la Revista Repertorio Americano, en su tercera época, surjan a partir de estos resultados y de la construcción del corpus. Las posibilidades son vastas: la poesía, la crítica literaria, lo femenino, los nuevos escritores y sus primicias, las preocupaciones pedagógicas, las vanguardias, las problemáticas universitarias, las recensiones y noticias de libros, las preocupaciones políticas, son algunos temas posibles de rastrear por otras personas investigadoras interesadas.

El ejercicio investigativo de cuyo resultado da cuenta este artículo, rinde homenaje a todas aquellas personas, presentes físicamente o en nuestra memoria, que contribuyeron a que Repertorio Americano volviera a circular, desde la maravillosa década de grandes luchas y militancias de 1970 hasta nuestros días, en la era digital en el siglo XXI, convertida en revista académica en el quehacer de una universidad latinoamericana.

\section{FUENTES}

Entrevista a Mendoza, Rolando, Miembro del primer consejo de redacción de la Revista. San Isidro de Heredia, 6 de diciembre de 2012.

Entrevista a Morales Hernández, Francisco, Gestor de la idea de publicación de la revista. Miembro del Consejo de Redacción. Miembro honorario del Consejo Editorial, Heredia, 7, 21 y 28 de febrero de 2013.

Entrevista a Picado de Bonilla, María Rosa, Primera co-directora de la Revista. Actual miembro honorario del Consejo Editorial. Los Yoses, San José, 4 de diciembre de 2012.

\section{REFERENCIAS}

Araya Pochet, Carlos. La Universidad Nacional y la educación superior estatal en veinte años de historia (1973-1993). Heredia: Colección Guayabo, Universidad Nacional de Costa Rica, 1994.

Azofeifa, Isaac Felipe. "En este número". Repertorio Americano, Vol.: 2 No. 2 (1975): 3.

Azofeifa, Isaac Felipe. “Tercera salida del Repertorio". Repertorio Americano, Vol.: 1 No. 1 (1974): 3-4. 
Beigel, Fernanda. "Las revistas culturales como documentos de la histórica latinoamericana". Utopía y Praxis Latinoamericana, Vol.: 8 No. 20 (2003): 105-115.

Crespo, Regina. Revistas en América Latina: proyectos literarios, políticos y culturales. México: Colección Miradas del Centauro, UNAM, 2010.

Galina Russell, Regina. Los nuevos retos de las revistas digitales académicas. En Actas del Simposio Internacional Nuevos retos de la publicación científica en Costa Rica. Universidad de Costa Rica. 6, 7, 8 de octubre 2010. Vicerrectoría de Investigación: Proyecto Latindex.

García Carrillo, Eugenio. Acta No. 94, Comisión Ad-hoc, SIA. Heredia: 1974.

García Monge, Joaquín. “El Otro Repertorio”. Repertorio Americano, Vol.: VII No. 1 (1924): 39.

España Calderón, Olmedo. (Comp.), Universidad Nacional. Historia y utopía. Heredia: Editorial Fundación UNA, 1998.

Instituto de Estudios Latinoamericanos. Repertorio Americano. Heredia: Universidad Nacional, 1974-1983.

Morales Hernández, Francisco. “¿Por qué Repertorio Americano en la Universidad Nacional?". Repertorio Americano, Vol.: 1 No. 1 (1974): 7-9.

Núñez Vargas, Benjamín, Hacia la universidad necesaria, Heredia: Depto. de Publicaciones, 1974.

Núñez Vargas, Benjamín. "Una universidad de excelencia”. En Universidad Nacional. Historia y utopía, compilado por Olmedo España. Heredia: Editorial Fundación Universidad Nacional, 1998.

Oliva Medina, Mario. España desde lejos. Intelectuales y letras centroamericanas sobre la guerra civil española (1931-1953). San José: Editorial de la Universidad Estatal a Distancia, 2011.

Oliva Medina, Mario. "Revista Repertorio Americano. Algunos alcances sobre su trayectoria, 1919-1958". Revista Izquierdas, Año 1 No. 1 (2008): 1-22.

Ovares, Flora. “Crónicas de lo efímero”. Revistas literarias de Costa Rica. (2011).

Pita, Alexandra. "Revistas culturales y redes intelectuales: una aproximación metodológica". En el Coloquio Internacional. La revista política en América Latina: el compromiso de la intelectualidad. Mimeo. 2012.

Pita, Alexandra y Carlos Marichal. Pensar el antiimperialismo. Ensayos de historia intelectual latinoamericana, 1900-1930. México: COLMEX/Universidad de Colima, 2011.

Ramírez, Celedonio. "La función de la nueva universidad". Repertorio Americano Vol.: 1, Año 2, (1975):1-8.

Ricoeur, Paul. La memoria, la historia el olvido. Madrid: Trotta, 2003.

Sistema Institucional de Archivo de la Universidad Nacional de Costa Rica. SIA. Heredia: Actas de la Comisión Ad-hoc.

Soto, Marybel. Roberto Brenes Mesén en el Repertorio Americano: contribuciones al pensamiento político costarricense". Tesis de Pregrado en licenciatura en Estudios Latinoamericanos, Universidad Nacional de Costa Rica, 1998.

Zelaya, Chéster. Acta No. 124, Comisión ad-hoc de la Universidad Nacional. Heredia: 24 de setiembre de 1974 . 
Zeledón, Rodrigo, Acta No. 55, Comisión ad-hoc de la Universidad Nacional, Heredia, 18 de setiembre de 1973 .

\begin{tabular}{|l|} 
\\
\hline $\begin{array}{l}\text { Soto Ramírez, Marybel. "El Repertorio Americano (1974-1983): Primera revista } \\
\text { académica fundada en la Universidad Nacional de Costa Rica". Revista Historia } \\
\text { de la Educación Latinoamericana. Vol. } 15 \text { No, 20, (2013): } 151 \text { - } 174 .\end{array}$ \\
\hline
\end{tabular}

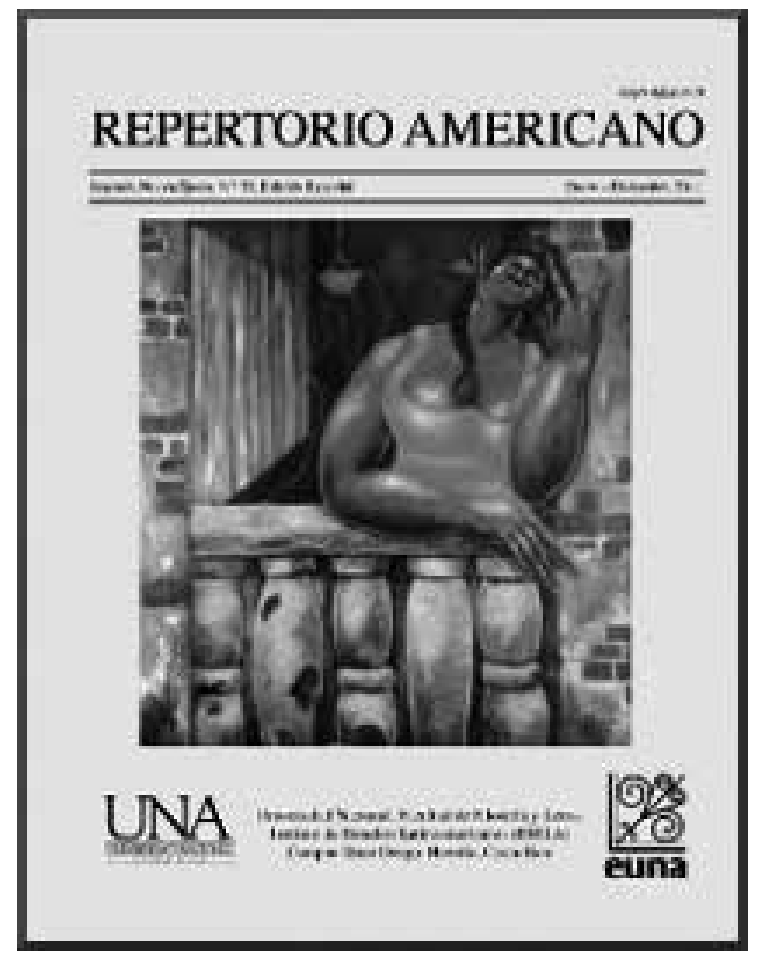

